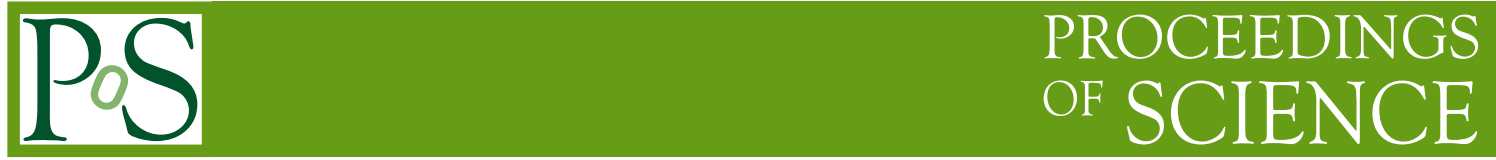

\title{
Heavy flavored probes of new physics with the ATLAS detector
}

Konstantinos Karakostas*

on behalf of the ATLAS collaboration

NTU Athens

E-mail: Konstantinos.Karakostasdcern.ch

Precision determinations of the flavor sector allow the indirect search of new physics effects. The ATLAS detector results and future potential in this sector are discussed, with particular emphasis on the search for rare $B_{s}$ and $B_{d}$ decays and the measurement of the phase $\phi_{s}$.

Xth Quark Confinement and the Hadron Spectrum,

October 8-12, 2012

TUM Campus Garching, Munich, Germany

${ }^{*}$ Speaker. 


\section{Introduction}

The ATLAS detector [ $[$ ] located at the Large Hadron Collider (LHC) [] is designed as a general purpose detector including a dedicated b-physics program. The b-physics program concentrates on low momentum di-muon signatures which can be efficiently triggered at an affordable event rate. In this article the focus is set on the measurement of the mixing phase for the $B_{s}^{0} \rightarrow J / \psi \phi$ decay and the search for rare decays studying $B_{s}^{0} \rightarrow \mu^{+} \mu^{-}$.

\subsection{ATLAS detector}

ATLAS is composed of several subdetectors with different functionalities. The Inner Detector (ID) records hits of charged particles, which are used to determine particle momenta and decay vertices. The tracker consists of three subsystems, the pixel detector, the silicon strip detector surrounded by the transition radiation tracker and is immersed in a $2 \mathrm{~T}$ magnetic field. The Muon Spectrometer (MS) provides a muon trigger system and a precise measurement of the muon momentum having dedicated tracking chambers and is in a toroidal magnetic field, a unique feature of ATLAS. Tracking performance measurements [3] provide us with a relative resolution of the transverse momentum $\sigma\left(p_{T}\right) / p_{T}$ of the order of $0.05 \%$, while for the low $p_{T}$ di-muons the mass resolution is varying with pseudorapidity $(\eta)$. The ID covers the $\eta$ range up to $|\eta|=2.5$ and the MS up to $|\eta|=2.7$. During 2011 ATLAS recorded $5.6 \mathrm{fb}^{-1}$ of data from p-p collisions at a center of mass energy $\sqrt{s}=7 \mathrm{TeV}$, while in 2012 about $22 \mathrm{fb}^{-1}$ at $\sqrt{s}=8 \mathrm{TeV}$.

\subsection{Search for New Physics}

The study of leptonic and semi-leptonic decays of $b$ hadrons plays an important part in the b-physics program of the ATLAS experiment, however purely leptonic decays (e.g. $B \rightarrow \mu \mu$ ) are highly suppressed in the Standard Model (SM) being forbidden at tree level and only possible through loop diagrams. New Physics (NP) processes, may manifest in the loops. Therefore, rare decays are excellent benchmark channels for probing NP effects. Measuring their properties may give indirect evidence for physics beyond the SM, or constrain the parameter space of new theoretical models.

The study of the $B_{s}^{0} \rightarrow J / \psi \phi$ decay is of significant interest to particle physics as it allows the measurement of the $B_{s}^{0}$ mixing phase, which is responsible for CP violation in this decay mode. The SM prediction for this CP violating phase is small, of the order of $\mathscr{O}\left(10^{-2}\right)$, so any measured excess would be a clear indication of New Physics entering the $B_{s}^{0}$ system.

\section{Measurement of $B_{s}^{0} \rightarrow J / \psi \phi$}

Flavour change via the weak interaction gives rise to $B_{s}^{0}-\bar{B}_{s}^{0}$ mixing, and consequently, the $B_{s}^{0}$ meson is found in the quantum superposition states denoted as "heavy" and "light", $\left(B_{s}^{H}\right.$ and $\left.B_{s}^{L}\right)$, which are CP eigenstates, odd and even respectively. These states have different masses and lifetimes. The oscillation frequency of $B_{s}^{0}$ meson mixing is characterized by the mass difference $\Delta m_{s}$ of the $B_{s}^{H}$ and $B_{s}^{L}$ mass eigenstates and by the CP-violating phase $\phi_{s}$ which arises from the interference between the decay and the mixing.

The $B_{s}^{0} \rightarrow J / \psi \phi$ decay has the peculiarity of a pseudo-scalar going into a pair of vector mesons. The vector mesons can have an even or odd relative orbital momentum $L$ in the final 
state $(L=0,1,2)$. Due to total angular momentum conservation the final state is therefore an admixture of CP-even $(L=0,2)$ and CP-odd $(L=1)$ states. Consequently, the decay is described by three time-dependent complex amplitudes each corresponding to a polarisation state of the vector mesons. The CP states are statistically separated through the time dependence of the decay and angular correlations in the final state particles [䧃]. A triplet of angular coordinates $\Omega=\left(\psi_{T}, \theta_{T}, \phi_{T}\right)$, defined in the transversity basis, can uniquely identify the angular signature of the decay.
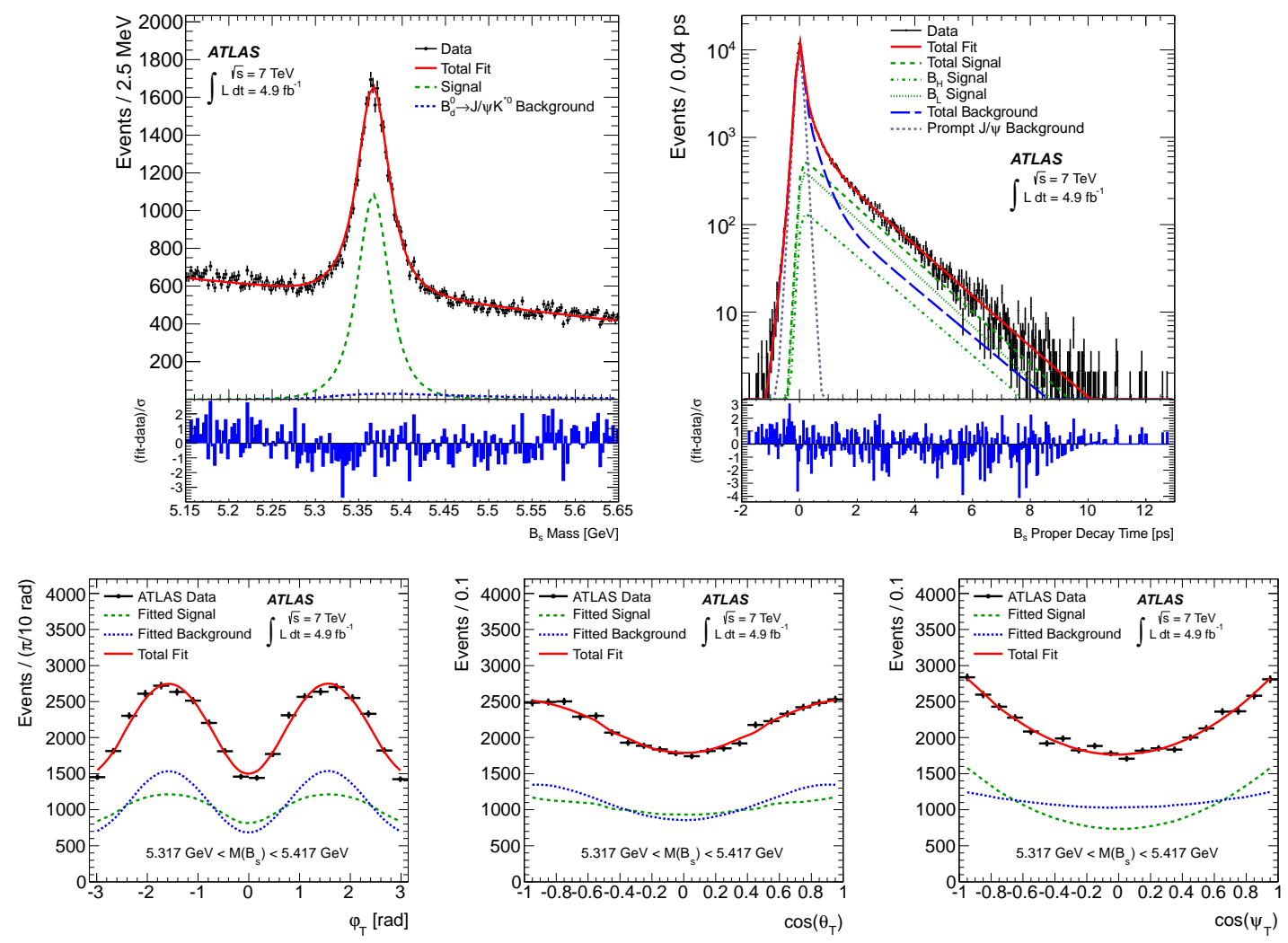

Figure 1: Top: (Left) Mass fit projection for the $B_{s}^{0}$. (Right) Proper decay time fit projection for the $B_{s}^{0}$. The pull distributions at the bottom show the difference between data and fit value normalized to the data uncertainty. Bottom: Fit projections for transversity angles. (Left): $\phi_{T},\left(\right.$ Centre): $\cos \theta_{T}$, (Right): $\cos \psi_{T}$.

For the measurement of the mixing phase $\phi_{s}$ for the $B_{s}^{0} \rightarrow J / \psi \phi$ decay, $4.9 \mathrm{fb}^{-1}$ of 2011 data with single and di-muon triggers were used, having the $p_{T}$ thresholds varying from $4 \mathrm{GeV}$ up to $10 \mathrm{GeV}$ depending on the trigger. Events found in good quality data with fully operational muon, tracking and trigger systems, are required to pass the selection criteria, for which a complete list can be found in [四].

Acceptance and background optimization were evaluated using large samples of signal and background Monte Carlo (MC) events with no $p_{T}$ cut applied on the generator level. In order to take into account the varying trigger configurations during data taking, the $\mathrm{MC}$ events were weighted to have the same trigger compositions as the data. The selection efficiency was measured in data and MC using data-driven procedures, like tag and probe method.

The measurement is based on a 5-dimensional, unbinned, maximum likelihood simultaneous fit to the distributions of mass, proper-time and angular coordinates $\left(\psi_{T}, \theta_{T}, \phi_{T}\right)$. The full fit con- 


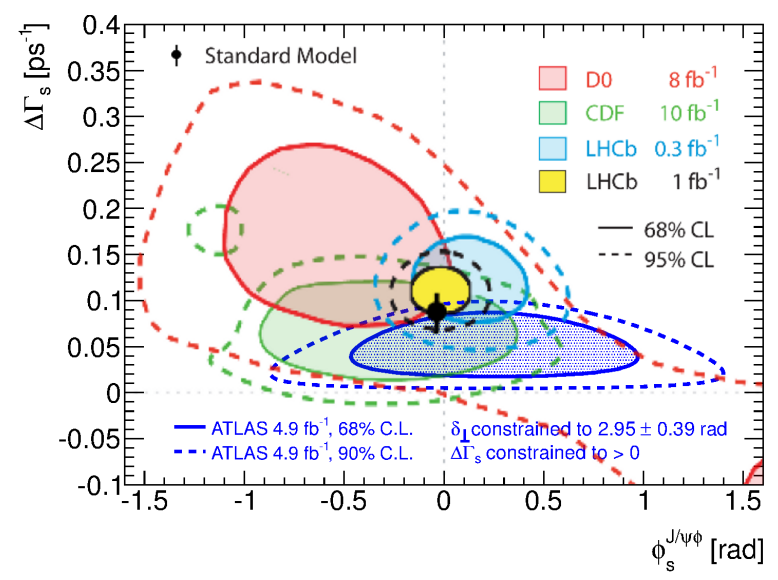

Figure 2: Likelihood contours in the $\phi_{S}-\Delta \Gamma_{S}$ plane. The two contours show the $68 \%$ and $95 \%$ confidence intervals (statistical errors only). The probability density function contains a fourfold ambiguity. Three minima are excluded by applying the constraints from the LHCb measurements. The black point corresponds to the Standard Model value.

tains 26 free parameters, 8 of them are the physics parameters we are mainly interested in and five parameters related to the amplitudes describing the proper time distribution of the $B_{s}^{0}$ decay (more details can be found in [四]). Results of this fit can be seen in Figure $\mathbb{W}$ through the projections of the distributions of mass, proper decay time and angles.

Likelihood contour plots in Figure $\square$ reveal consistency with SM and in terms of $\phi_{S}$ a precision similar to Tevatron experiments [1] while in terms of $\Delta \Gamma_{S}$ comparable to $\mathrm{LHCb}$ [6].

\begin{tabular}{c||c|c|c} 
Parameter & Value & Statistical uncertainty & Systematic uncertainty \\
\hline \hline$\phi_{S}(\mathrm{rad})$ & 0.22 & 0.41 & 0.10 \\
$\Delta \Gamma_{S}\left(\mathrm{ps}^{-1}\right)$ & 0.053 & 0.021 & 0.010 \\
$\Gamma_{S}\left(\mathrm{ps}^{-1}\right)$ & 0.677 & 0.007 & 0.004 \\
$\left|A_{0}(0)\right|^{2}$ & 0.528 & 0.006 & 0.009 \\
$\left|A_{\|}(0)\right|^{2}$ & 0.220 & 0.008 & 0.007
\end{tabular}

Table 1: Fitted values for the parameters along with their statistical and systematic uncertainties [䧃]. The amplitudes $A_{0}(0)$ and $A_{\|}(0)$ are for the CP-even components of the $B_{s}^{0} \rightarrow J / \psi \phi$ decay. $A_{\perp}(0)$ is the CP-odd amplitude. They have corresponding strong phases $\delta_{0}, \delta_{\|}$and $\delta_{\perp}$; by convention $\delta_{0}$ is set to be zero. The S-wave amplitude $A_{S}(0)$ gives the fraction of $B_{s}^{0} \rightarrow J / \psi K^{+} K\left(f_{0}\right)$ and has a related strong phase $\delta_{S}$.

Concluding on the $B_{s}^{0} \rightarrow J / \psi \phi$, without any flavor tagging, there is strong correlations between the two strong phases, $\delta_{\|}$and $\delta_{\perp}$ [ [母]. Both parameters cannot be fitted simultaneously. In the fit, the strong phase $\delta_{\perp}$, was constrained to $\delta_{\perp}=2.95 \pm 0.39$ from the LHCb measurement [ $[\mathbf{D}]$. The second strong phase $\delta_{\|}$, was fitted very close to its symmetry point at $\pi$ resulting in a highly non-gaussian likelihood shape around the minimum. Results of this fit are given in Table 1.

\section{Search for $B_{s}^{0} \rightarrow \mu^{+} \mu^{-}$decay}

The $B_{s}^{0} \rightarrow \mu^{+} \mu^{-}$decay is a Flavor Changing Neutral Current process involving a $b \rightarrow s$ quark transition. It is strongly suppressed in the SM and occurs at the lowest order through one-loop 
diagrams, with a theoretical prediction of its branching ratio to $(3.2 \pm 0.2) \times 10^{-9}$ [ [8]. Contributions from New Physics could significantly increase this branching ratio.
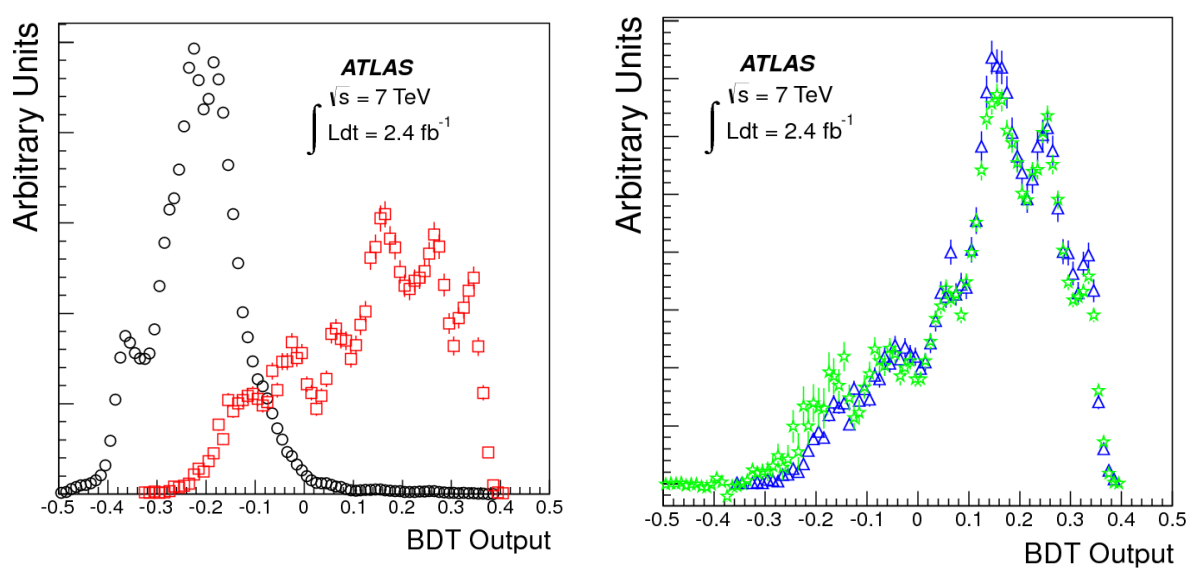

Figure 3: Distributions of the response of the BDT classifier. Left: $B_{s}^{0} \rightarrow \mu^{+} \mu^{-} \mathrm{MC}$ sample (squares) and data sidebands (circles). Right: $B^{ \pm} \rightarrow J / \psi K^{ \pm}$events from MC samples (triangles) and sideband-subtracted data (stars).

For this measurement [Q], a di-muon trigger with $p_{T}>4 \mathrm{GeV}$ for each of the two muon candidates was used and the measurement of the branching fraction performed with respect to an abundant decay in order to be independent of the uncertainties of the luminosity, $b \bar{b}$ production cross-section measurements and to minimize systematic uncertainties. The $B_{s}^{0} \rightarrow \mu^{+} \mu^{-}$branching fraction is measured with respect to the reference channel $B^{ \pm} \rightarrow J / \psi K^{ \pm} \rightarrow \mu^{+} \mu^{-} K^{ \pm}$that has a large production rate and a similar (di-muon) final state. Similar selection cuts are applied to both signal and reference channel, in order to keep systematic uncertainties low. Assuming the branching ratio of $B_{s}^{0} \rightarrow \mu^{+} \mu^{-}$the probability of signal to be observed is low and therefore an upper limit on its value will be given. The branching fraction is computed as:

$$
B R\left(B_{s}^{0} \rightarrow \mu^{+} \mu^{-}\right)=\frac{N_{\mu^{+} \mu^{-}}}{N_{J / \psi K^{ \pm}}} \times \frac{\alpha_{J / \psi K^{ \pm}} \cdot \varepsilon_{J / \psi K^{ \pm}}}{\alpha_{B_{s} \rightarrow \mu^{+} \mu^{-}} \cdot \varepsilon_{B_{s} \rightarrow \mu^{+} \mu^{-}}} \times \frac{f_{u}}{f_{s}} \times B R\left(B^{ \pm} \rightarrow J / \psi K^{ \pm} \rightarrow \mu^{+} \mu^{-} K^{ \pm}\right)
$$

The right-hand-side of 1 . Dincludes: the branching fraction of the reference channel the relative production cross sections for $B^{ \pm}$and $B_{s}^{0}\left(f_{u} / f_{s}=0.267 \pm 0.021\right.$ assuming $f_{u}=f_{d}$ [ए]] and no $p_{T}$ or $\eta$ dependence), acceptance and efficiency ratios estimated from MC samples; event yields for measured and reference channel.

The signal region, defined as a di-muon invariant mass region of $\pm 300 \mathrm{MeV}$ around the $B_{s}^{0}$ mass, is excluded from the optimization of the selection procedure (blind analysis). Instead, the $B_{s}^{0} \rightarrow \mu^{+} \mu^{-} \mathrm{MC}$ signal and the background extrapolated from sidebands (4766 - $5066 \mathrm{MeV}$ and $5666-5966 \mathrm{MeV}$ ) are used. To avoid any bias in the optimization procedure, the data sample is split into two parts: one half of the data is used for the selection optimization and the other half for the background measurement. The number of signal candidates $N_{\mu^{+}} \mu^{-}$is counted after unblinding. The yield $N_{J / \psi K^{ \pm}}$is measured with a maximum likelihood fit on the selected events, assessing the systematic uncertainties by varying the bin size, the signal and background fit models and by the inclusion of the per-event mass resolution into the fit. The upper limit is extracted with 
the modified frequentist method (CLs) [Ш]]. In order to exploit the best di-muon mass resolution the data sample was split into three mass resolution categories defined by the larger pseudorapidity value $|\eta|_{\max }$ of the two muons in each event. The three categories were defined by the intervals $|\eta|_{\max }=0-1,1-1.5$ and $1.5-2.5$. The event selection was optimized separately for each category and the results are combined within the CLs method.
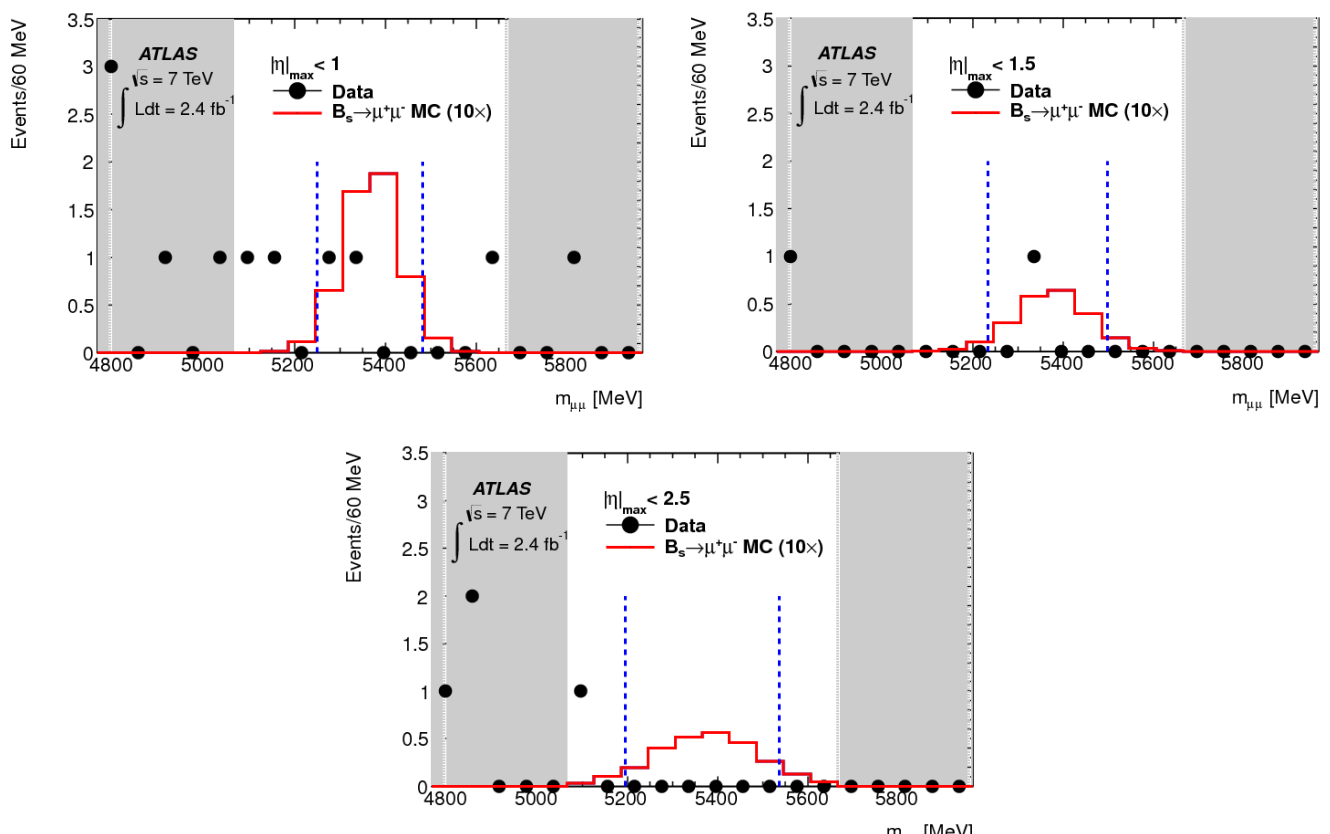

Figure 4: Invariant mass distribution of $B_{s}^{0} \rightarrow \mu^{+} \mu^{-}$candidates. For each mass-resolution category $\left(|\eta|_{\max } \leq 1, \leq 1.5, \leq 2.5\right)$ each plot shows the invariant mass distribution for the selected candidates in data (dots), the signal (continuous line) as predicted by MC scaled by a factor 10 for the visualization and assuming $\operatorname{BR}\left(B_{s}^{0} \rightarrow \mu^{+} \mu^{-}\right)=3.5 \times 10^{-8}$, and two dashed vertical lines correspond to the optimized $\Delta_{m}$ cut. The grey areas correspond to the sidebands used in the analysis.

The background has continuous and resonant components. The continuous background originates from the random combination of muon tracks created in $q \bar{q}$ annihilation processes which could be of prompt (e.g. Drell-Yan) or of non-prompt (dominated by $b \bar{b} \rightarrow \mu \mu X$ decays) origin. The resonant background comes from the decays of neutral B mesons with one $(B \rightarrow K \mu v)$ or two hadrons $\left(B \rightarrow h h\right.$ like $\left.B_{(s)} \rightarrow \pi \pi, K K, \pi K\right)$ in the final state, for which one or both hadrons are mis-identified as muons. The resonant background mimics the signal topology and therefore is hard to suppress; its contribution was estimated from dedicated MC samples using data-driven mis-identification rates.

For the selection of the $B_{s}^{0}$ candidates out of the continuum background, 14 discriminating variables were identified as inputs for a multi-variate analysis [ $[$ ]. The most discriminating variables are: the distance of the $B_{s}^{0}$ decay (secondary) vertex from the primary vertex, the proper time significance, the pointing angle (absolute value of the angle between $\Delta \vec{x}$ and $\vec{p}^{B}$ in the transverse plane, where the vector $\Delta \vec{x}$ is a vector from the primary vertex (PV) to the secondary vertex (SV) and the PV is chosen as the closest in $z$ to the $\mathrm{SV}$ of the candidate), the isolation of the di-muon tracks and the transverse momentum of the $B_{s}^{0}$. The implementation of the Boosted Decision Trees (BDT) method in the Toolkit for Multivariate Data Analysis (TMVA) package [12] has been found 
to be the most powerful multi-variate analysis tool. The BDT output separates well the signal and the background, as seen in Figure B (left). A crosscheck on the $B^{ \pm}$reference channel (by applying the same BDT weights) shows good agreement between the MC signal and the sideband-subtracted data ( Figure B, right).

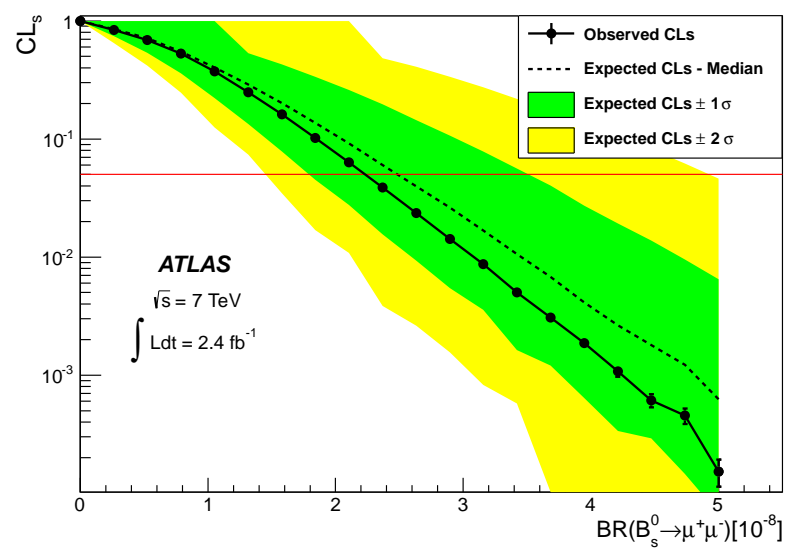

Figure 5: Observed CLs (circles) as a function of $\mathrm{BR}\left(B_{s}^{0} \rightarrow \mu^{+} \mu^{-}\right)$. The 95\% CL limit is indicated by the horizontal (red) line. The dark (green) and light (yellow) bands correspond to $\pm 1 \sigma$ and $\pm 2 \sigma$ fluctuations on the expectation (dashed line), based on the number of the observed events in the signal and sideband regions.

The inputs to the limit extraction are the observed number of events in the three resolution regions $(2 / 1 / 0)$; the events observed in the half of the sideband region $(5 / 0 / 2)$, giving a total of 6.1 events expected in the signal region; the contribution of the resonant background $(0.24$ events in total). The expected limit is calculated prior to unblinding in the background-only hypothesis and is found to be $2.3_{-0.5}^{+1.0} \times 10^{-8}$ at $95 \% \mathrm{CL}$. The corresponding observed limit is $2.2 \times 10^{-8}$ at 95\% CL [Q], shown in Figure 1 .
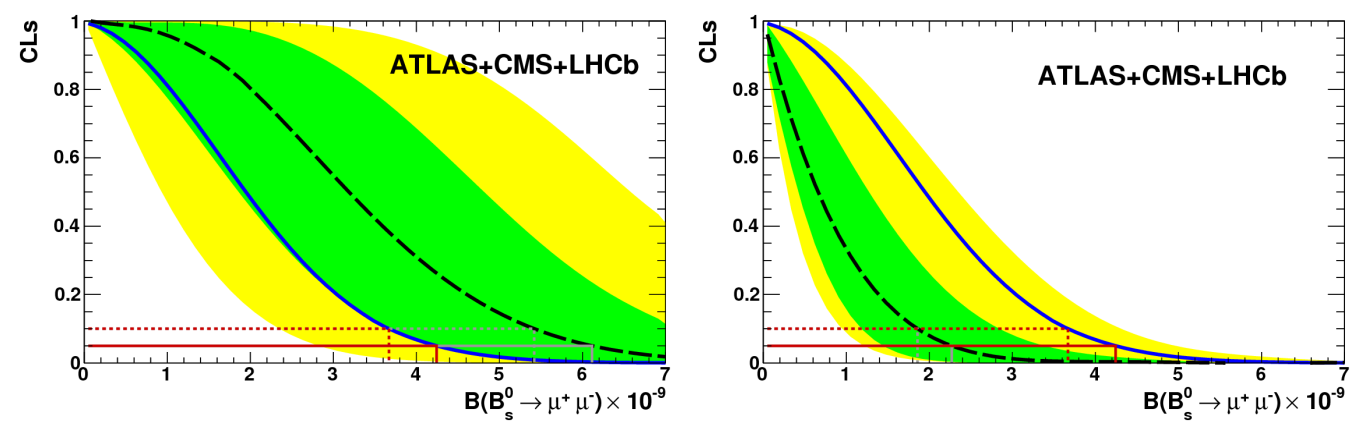

Figure 6: Observed CLs (continuous line) as a function of $\operatorname{BR}\left(B_{s}^{0} \rightarrow \mu^{+} \mu^{-}\right)$with the ATLAS+CMS+LHCb experiments. The $95 \%$ CL limit is indicated by the horizontal (red) line. The dark (green) and light (yellow) bands correspond to $\pm 1 \sigma$ and $\pm 2 \sigma$ fluctuations on the expectation (dashed line) for background and SM signal (left plot) and background-only (right plot).

\section{Combination of the limits for $B_{s}^{0} \rightarrow \mu^{+} \mu^{-}$at the LHC}

The ATLAS analysis has been combined with the equivalent analysis of the CMS [1D2] and LHCb 


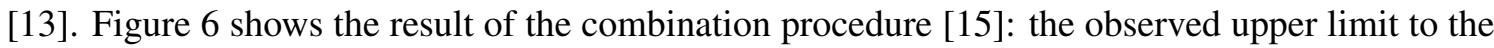
branching fraction is $4.2 \times 10^{-9}$ at $95 \% \mathrm{CL}$. The combined number of observed event is compatible with the expected background plus a SM signal within $1 \sigma\left(1-C L_{S+b}=84 \%\right)$ (Figure 6 left), while the $\mathrm{p}$-value of the background-only hypothesis is $1-C L_{b}=5 \%$ (Figure 6 right).

\section{Conclusion}

ATLAS has a rich b-physics program which includes indirect searches for new physics, such as the rare decay $B_{s}^{0} \rightarrow \mu^{+} \mu^{-}$, and measurements of CP-violating phase in the $B_{s}^{0} \rightarrow J / \psi \phi$ decay. An upper limit on the branching fraction of $B_{s}^{0} \rightarrow \mu^{+} \mu^{-}$of $2.2 \times 10^{-8}$ at $95 \%$ CL has been set using $2.4 \mathrm{fb}^{-1}$ of 2011 data. Several parameters describing the $B_{s}^{0}$ meson were measured, with $4.9 \mathrm{fb}^{-1}$ of 2011 data, and their values are consistent with the theoretical expectations. In particular the CP-violating phase $\phi_{s}$ for the $B_{s}^{0}$ meson was measured to be $0.22 \pm 0.41$ (stat.) \pm 0.10 (syst.) rad.

\section{Acknowledgements}

This presentation was co-funded by the European Union (European Social Fund ESF) and Greek national funds through the Operational Program "Education and Lifelong Learning" of the National Strategic Reference Framework (NSRF).

\section{References}

[1] ATLAS Collaboration, JINST, 3S08003 (2008)

[2] L. Evans and P. Bryant, JINST 3 S08001 (2008)

[3] ATLAS Collaboration, ATLAS-CONF-2011-046, (2011)

[4] ATLAS Collaboration, JHEP 12, 072 [arXiv:1208.0572] (2012)

[5] CDF Collaboration, Phys. Rev. Lett. 107:272001(2011); D0 Collaboration, Phys. Rev. D 85,032006 (2012)

[6] LHCb Collaboration, CONF-2012-002 (2012)

[7] LHCb Collaboration, Phys. Rev. Lett. 108, 101803 (2012)

[8] A. J. Buras et al., JHEP 1010009 [arXiv:1005.5310] (2010)

[9] ATLAS Collaboration, Phys. Lett. B713 180-196 [arXiv:1204.0735] (2012)

[10] LHCb Collaboration, Phys. Rev. D85 (2012) 032008. [arXiv:1111.2357]; Averages of b-hadron, c-hadron, and $\tau$-lepton Properties, [arXiv:1010.1589].

[11] T. Junk, Nucl. Instrum. Meth. A434 (1999) 435; A. Read, J. Phys. G28 2693 (2002)

[12] TMVA 4, Toolkit for Multivariate Data Analysis with ROOT, CERN, [arXiv:physics/0703039]

[13] CMS Collaboration, JHEP 1204 (2012) 033, [arXiv:1203.3976].

[14] LHCb Collaboration, Phys. Lett. B699 (2011) 330, [arXiv:1103.2465]; Phys. Rev. Lett. 108 (2012) 231801, [arXiv:1203.4493]

[15] The ATLAS, CMS and LHCb Collaborations, Search for the decays $B_{(s)}^{0} \rightarrow \mu^{+} \mu^{-}$with the ATLAS, CMS and LHCb experiments, ATLAS-CONF-2012-017, CMS-PAS-BPH-12-2009, LHCb-CONF-2012-017, June 2012 